\title{
Biomechanical effect of pedicle screw distribution in AIS instrumentation using a segmental translation technique: computer modeling and simulation
}

Xiaoyu Wang ${ }^{1,2}$, A. Noelle Larson ${ }^{3}$, Dennis G. Crandall ${ }^{4}$, Stefan Parent ${ }^{2}$, Hubert Labelle ${ }^{2}$, Charles G. T. Ledonio ${ }^{5}$ and Carl-Eric Aubin ${ }^{1,2^{*}}$

\begin{abstract}
Background: Efforts to select the appropriate number of implants in adolescent idiopathic scoliosis (AIS) instrumentation are hampered by a lack of biomechanical studies. The objective was to biomechanically evaluate screw density at different regions in the curve for AIS correction to test the hypothesis that alternative screw patterns do not compromise anticipated correction in AIS when using a segmental translation technique.

Methods: Instrumentation simulations were computationally performed for 10 AIS cases. We simulated simultaneous concave and convex segmental translation for a reference screw pattern (bilateral polyaxial pedicle screws with dorsal height adjustability at every level fused) and four alternative patterns; screws were dropped respectively on convex or concave side at alternate levels or at the periapical levels ( 21 to $25 \%$ fewer screws). Predicted deformity correction and screw forces were compared.

Results: Final simulated Cobb angle differences with the alternative screw patterns varied between $1^{\circ}$ to $5^{\circ}$ (39 simulations) and $8^{\circ}(1$ simulation) compared to the reference maximal density screw pattern. Thoracic kyphosis and apical vertebral rotation were within $2^{\circ}$ of the reference screw pattern. Screw forces were $76 \pm 43 \mathrm{~N}, 96 \pm 58$ $\mathrm{N}, 90 \pm 54 \mathrm{~N}, 82 \pm 33 \mathrm{~N}$, and $79 \pm 42 \mathrm{~N}$, respectively, for the reference screw pattern and screw dropouts at convex alternate levels, concave alternate levels, convex periapical levels, and concave periapical levels. Bone-screw forces for the alternative patterns were higher than the reference pattern $(p<0.0003)$. There was no statistical bone-screw force difference between convex and concave alternate dropouts and between convex and concave periapical dropouts $(p>0.28)$. Alternate dropout screw forces were higher than periapical dropouts $(p<0.05)$.

Conclusions: Using a simultaneous segmental translation technique, deformity correction can be achieved with $23 \%$ fewer screws than maximal density screw pattern, but resulted in 25\% higher bone-screw forces. Screw dropouts could be either on the convex side or on the concave side at alternate levels or at periapical levels. Periapical screw dropouts may more likely result in lower bone-screw force increase than alternate level screw dropouts.
\end{abstract}

Keywords: Pedicle screw, Adolescent idiopathic scoliosis, Instrumentation, Biomechanical modeling, Simulation, Screw pattern, Screw density, Screw distribution

\footnotetext{
* Correspondence: carl-eric.aubin@polymtl.ca

'Department of Mechanical Engineering, Polytechnique Montréal, P.O. Box

6079, Downtown Station, Montreal, Quebec H3C 3A7, Canada

${ }^{2}$ Sainte-Justine University Hospital Center, 3175, Cote Sainte-Catherine Road,

Montreal, Quebec H3T 1C5, Canada

Full list of author information is available at the end of the article
}

(c) The Author(s). 2017 Open Access This article is distributed under the terms of the Creative Commons Attribution 4.0 International License (http://creativecommons.org/licenses/by/4.0/), which permits unrestricted use, distribution, and reproduction in any medium, provided you give appropriate credit to the original author(s) and the source, provide a link to the Creative Commons license, and indicate if changes were made. The Creative Commons Public Domain Dedication waiver (http://creativecommons.org/publicdomain/zero/1.0/) applies to the data made available in this article, unless otherwise stated. 


\section{Background}

Pedicle screw fixation has become the state-of-the-art instrumentation for the surgical treatment of adolescent idiopathic scoliosis (AIS), resulting in better deformity correction and lower revision surgery rates compared to hybrid or hook-rod constructs [1-3]. However, wide variation in clinical practice persists regarding the number and distribution of pedicle screws used, as well as the surgical techniques for the treatment of pediatric scoliosis.

Screw density is defined as the number of screws per level fused. There may be multiple clinical and biomechanical factors in determining screw density and distribution. Certain screw types and distributions are required in order to perform specific correction maneuvers, such as apical vertebral derotation and segmental vertebral derotation [4]. The effect of screw density depends also on the construct design. Some screw types and patterns tended to overconstrain the instrumented spine generating high (overconstraining) bone-screw forces in highdensity screw constructs, such as monoaxial screws [5]; screws with multiple degrees of adjustability allowed the overconstraining effect to be reduced and segmental translation to be performed in a gradual and incremental way to lower the overall bone-screw force level $[5,6]$.

A structured literature review revealed that the mean reported screw density varies from 1.04 to 2.0 , whereas the average curve corrections only varied from 64 to $70 \%$ [7]. Some surgeons routinely use two screws at every level fused where other surgeons may use up to $46 \%$ fewer screws [8,9]. High screw density constructs have been associated with increased operative time, blood loss, radiation exposure, instrumentation costs, and risk of screw-related complications [10-14]. Constructs with fewer screws may have benefits for optimal use of health care resources [7, 8]. Some studies note improved percent correction of major coronal curve in the high screw density cohort $[8,9]$; but, in other studies, no significant difference in outcome was found between the high and low screw density groups $[10,15,16]$.

Until recently, studies of screw density have been underpowered, included hybrid constructs, or based on retrospective review of clinical data. Further, there is a lack of biomechanical data guiding screw number and placement. Thus, practice is mostly based on individual preferred technique, and scientific progress is limited by the inability to test alternative screw patterns on a given patient. In contrast to studies based on clinical data analysis, biomechanical studies using computerized patientspecific models allow the assessment and comparison of variable screw numbers and patterns with different correction techniques simulated for the same case. The objective of this study was to use computerized patientspecific spine models to biomechanically evaluate screw dropouts at different regions in the curve for AIS correction to test the hypothesis that alternative screw patterns do not compromise anticipated correction in AIS when using segmental translation as the primary correction technique.

\section{Methods}

Numerical simulations of posterior spinal instrumentations were performed using computerized patientspecific biomechanical models of 10 AIS patients in order to assess the effect of screw density on curve correction and bone-screw forces. With the institutional review board approval, the cases were randomly selected from AIS patients having undergone instrumented spinal fusion at our university hospital center during the last 6 years. Clinical indices are provided in Table 1. Modeling and simulation details are presented in the following subsections.

\section{Computerized patient-specific biomechanical spine model} Three-dimension (3D) spine geometry of the selected cases was built using calibrated preoperative coronal and lateral radiographs and $3 \mathrm{D}$ multi-view reconstruction techniques [17]. The process began with the identification of key anatomical landmarks of each vertebra, typically, the pedicles, vertebral endplate middle and corner points, and transverse and spinous process extremities. The 2D coordinates of these landmarks allowed the determination of their $3 \mathrm{D}$ coordinates in space, which was done using a self-calibration and optimization algorithm $[17,18]$. The reconstruction process was completed by registering detailed vertebral models using the 3D coordinates of the key landmarks and a free form deformation technique [17, 18]. Average accuracies for pedicles and vertebral bodies were $1.6 \mathrm{~mm}$ (SD $1.1 \mathrm{~mm}$ ) and $1.2 \mathrm{~mm}$ (SD $0.8 \mathrm{~mm}$ ), respectively [18].

Vertebrae from T1 through L5 and the pelvis were modeled as rigid parts which were connected with multiple flexible elements respectively representing the biomechanical effect of the intervertebral disc, anterior longitudinal ligament (ALL), posterior longitudinal ligament (PLL), ligamentum flavum (LF), intertransverse ligament (ITL), facet joint capsule (FC), and interspinous ligament (ISL) combined with supraspinous ligament (SSL). Six translational springs were used to respectively represent (1) ALL, (2) PLL, (3) LF, (4) left ITL, (5) right ITL, and (6) the combined effect of ISL and SSL. The biomechanical behavior of the facet joints is more complex compared to the other intervertebral ligamentous elements; they were respectively represented with a six-dimensional general spring [19]. A primary general spring was used to represent the intervertebral disc to which the effect of all elements and factors which were not explicitly modeled in this study was incorporated by 
Table 1 Clinical indices

\begin{tabular}{|c|c|c|c|c|c|c|c|c|c|c|c|}
\hline Case no. & & 1 & 2 & 3 & 4 & 5 & 6 & 7 & 8 & 9 & 10 \\
\hline Sex & & $\mathrm{F}$ & $\mathrm{F}$ & $\mathrm{F}$ & $\mathrm{F}$ & $\mathrm{F}$ & M & $\mathrm{F}$ & F & F & $F$ \\
\hline Age & & 14 & 16 & 19 & 17 & 14 & 15 & 16 & 15 & 15 & 14 \\
\hline Height $(\mathrm{cm}$ & & 154 & 162 & 162 & 168 & 170 & 172 & 165 & 170 & 159 & 148 \\
\hline Weight $(\mathrm{kg})$ & & 52 & 56 & 47 & 56 & 59 & 55 & 53 & 48 & 59 & 39 \\
\hline Lenke class & & $1 \mathrm{~A}$ & $1 \mathrm{~A}$ & $3 B$ & $4 \mathrm{~A}$ & $3 B$ & $3 C$ & $1 \mathrm{~A}$ & $1 \mathrm{~A}$ & $3 C$ & $2 \mathrm{~A}$ \\
\hline MT superio & vertebra & T6 & T6 & T5 & T5 & T5 & $\mathrm{T7}$ & T5 & T4 & T6 & T5 \\
\hline Apical verte & & T9 & T11 & T8 & T8 & T8 & $\mathrm{T} 10$ & T9 & T8 & T9 & T9 \\
\hline Inferior enc & ebra & $\mathrm{T} 12$ & L2 & T11 & $\mathrm{T} 11$ & $\mathrm{~T} 12$ & L1 & L1 & $\mathrm{T} 12$ & $\mathrm{~T} 12$ & $\mathrm{~T} 11$ \\
\hline PT Cobb & Preop. & $32^{\circ}$ & $31^{\circ}$ & $39^{\circ}$ & $52^{\circ}$ & $31^{\circ}$ & $34^{\circ}$ & $28^{\circ}$ & $9^{\circ}$ & $40^{\circ}$ & $42^{\circ}$ \\
\hline & Left bending & $22^{\circ}$ & $10^{\circ}$ & $12^{\circ}$ & $17^{\circ}$ & $24^{\circ}$ & $6^{\circ}$ & $20^{\circ}$ & $5^{\circ}$ & $15^{\circ}$ & $28^{\circ}$ \\
\hline & Right bending & $35^{\circ}$ & $41^{\circ}$ & $42^{\circ}$ & $54^{\circ}$ & $35^{\circ}$ & $37^{\circ}$ & $32^{\circ}$ & $20^{\circ}$ & $41^{\circ}$ & $46^{\circ}$ \\
\hline MT Cobb & Preop. & $55^{\circ}$ & $52^{\circ}$ & $58^{\circ}$ & $60^{\circ}$ & $64^{\circ}$ & $62^{\circ}$ & $44^{\circ}$ & $48^{\circ}$ & $67^{\circ}$ & $51^{\circ}$ \\
\hline & Left bending & $59^{\circ}$ & $60^{\circ}$ & $63^{\circ}$ & $64^{\circ}$ & $65^{\circ}$ & $62^{\circ}$ & $73^{\circ}$ & $55^{\circ}$ & $63^{\circ}$ & $60^{\circ}$ \\
\hline & Right bending & $29^{\circ}$ & $25^{\circ}$ & $29^{\circ}$ & $38^{\circ}$ & $50^{\circ}$ & $27^{\circ}$ & $10^{\circ}$ & $30^{\circ}$ & $35^{\circ}$ & $30^{\circ}$ \\
\hline MT AVR & Preop. & $16^{\circ}$ & $17^{\circ}$ & $18^{\circ}$ & $19^{\circ}$ & $19^{\circ}$ & $20^{\circ}$ & $19^{\circ}$ & $18^{\circ}$ & $22^{\circ}$ & $19^{\circ}$ \\
\hline TL/L Cobb & Preop. & $37^{\circ}$ & $37^{\circ}$ & $39^{\circ}$ & $30^{\circ}$ & $42^{\circ}$ & $48^{\circ}$ & $35^{\circ}$ & $39^{\circ}$ & $40^{\circ}$ & $32^{\circ}$ \\
\hline & Left bending & $2^{\circ}$ & $5^{\circ}$ & $20^{\circ}$ & $9^{\circ}$ & $5^{\circ}$ & $30^{\circ}$ & $10^{\circ}$ & $10^{\circ}$ & $9^{\circ}$ & $2^{\circ}$ \\
\hline & Right bending & $49^{\circ}$ & $50^{\circ}$ & $42^{\circ}$ & $40^{\circ}$ & $65^{\circ}$ & $50^{\circ}$ & $42^{\circ}$ & $45^{\circ}$ & $70^{\circ}$ & $49^{\circ}$ \\
\hline TL/L AVR & Preop. & $5^{\circ}$ & $3^{\circ}$ & $7^{\circ}$ & $7^{\circ}$ & $3^{\circ}$ & $11^{\circ}$ & $6^{\circ}$ & $11^{\circ}$ & $9^{\circ}$ & $3^{\circ}$ \\
\hline Kyphosis & Preop. & $7^{\circ}$ & $23^{\circ}$ & $37^{\circ}$ & $28^{\circ}$ & $22^{\circ}$ & $11^{\circ}$ & $20^{\circ}$ & $18^{\circ}$ & $7^{\circ}$ & $15^{\circ}$ \\
\hline Lordosis & Preop. & $42^{\circ}$ & $37^{\circ}$ & $27^{\circ}$ & $45^{\circ}$ & $33^{\circ}$ & $15^{\circ}$ & $47^{\circ}$ & $40^{\circ}$ & $30^{\circ}$ & $32^{\circ}$ \\
\hline UIV & & T4 & T4 & T4 & T3 & T3 & T4 & T4 & T4 & T3 & T3 \\
\hline LIV & & L2 & L3 & L1 & L1 & L1 & L2 & L2 & L2 & L2 & L1 \\
\hline
\end{tabular}

$F$ female, $M$ male, PT proximal thoracic, $M T$ main thoracic, $T L / L$ thoracolumbar/lumbar, $A V R$ apical vertebral rotation, UIV upper instrumented vertebra, LIV lower instrumented vertebra

introducing weighting factors to the diagonal elements of its stiffness matrix, e.g., the rib cage increased the stiffness of the thoracic spine by 40,35 , and $31 \%$ respectively in flexion/extension, lateral bending, and axial rotation [20]. The multibody modeling elements are illustrated in Fig. 1.

The six translational springs were modeled as cablelike elements on the computer-aided engineering platform, Adams/View, Version MD Adams 2010 (MSC Software Corporation, Santa Ana, CA, USA). Their stiffness in compression were set to null and those in traction were adapted to the reported experiment results on cadaveric specimens, e.g., $23.6 \mathrm{~N} / \mathrm{mm}$ (ALL), $24.9 \mathrm{~N} /$ $\mathrm{mm}$ (PLL), $32.6 \mathrm{~N} / \mathrm{mm}$ (LF), $12.9 \mathrm{~N} / \mathrm{mm}$ (ITL), and $32.1 \mathrm{~N} / \mathrm{mm}$ (ISL combined with SSL) [21-23] for T6-T7 functional spinal unit. Instrumented posterior spinal fusion with pedicle screw fixation involves the removal of the facet joint capsules and the spinous process. The biomechanical effects of pedicle screw placement surgical procedure were modeled by removing the facet joint capsule and the interspinous model elements, whose mechanical properties were calibrated using experiment data reported in the literature, i.e., osteotomies involved in pedicle screw placement procedure reduced the stiffness of a functional spine unit by $17 \%$ in axial rotation [24-27], 15\% sagittal plane flexion [24, 28, 29], 3.8\% in coronal plane bending [24], and $14 \%$ in axial compressive load [30-33]. The stiffness matrix of the primary general spring was calibrated such that the load-displacement simulations with the model of a functional spinal unit reproduced the reported loaddisplacement data [34-37]. All model element stiffness were further adjusted such that side bending simulations reproduced the Cobb angles measured on the patient's side bending radiographs using a similar optimization technique reported in $[38,39]$.

\section{Biomechanical modeling and simulations of spinal instrumentation}

Based on the actual instrumented fusion levels of the selected cases and the well-accepted alternative screw densities [4], five screw patterns were biomechanically evaluated on each case. The screw patterns were a reference screw pattern with bilateral screws at every level 


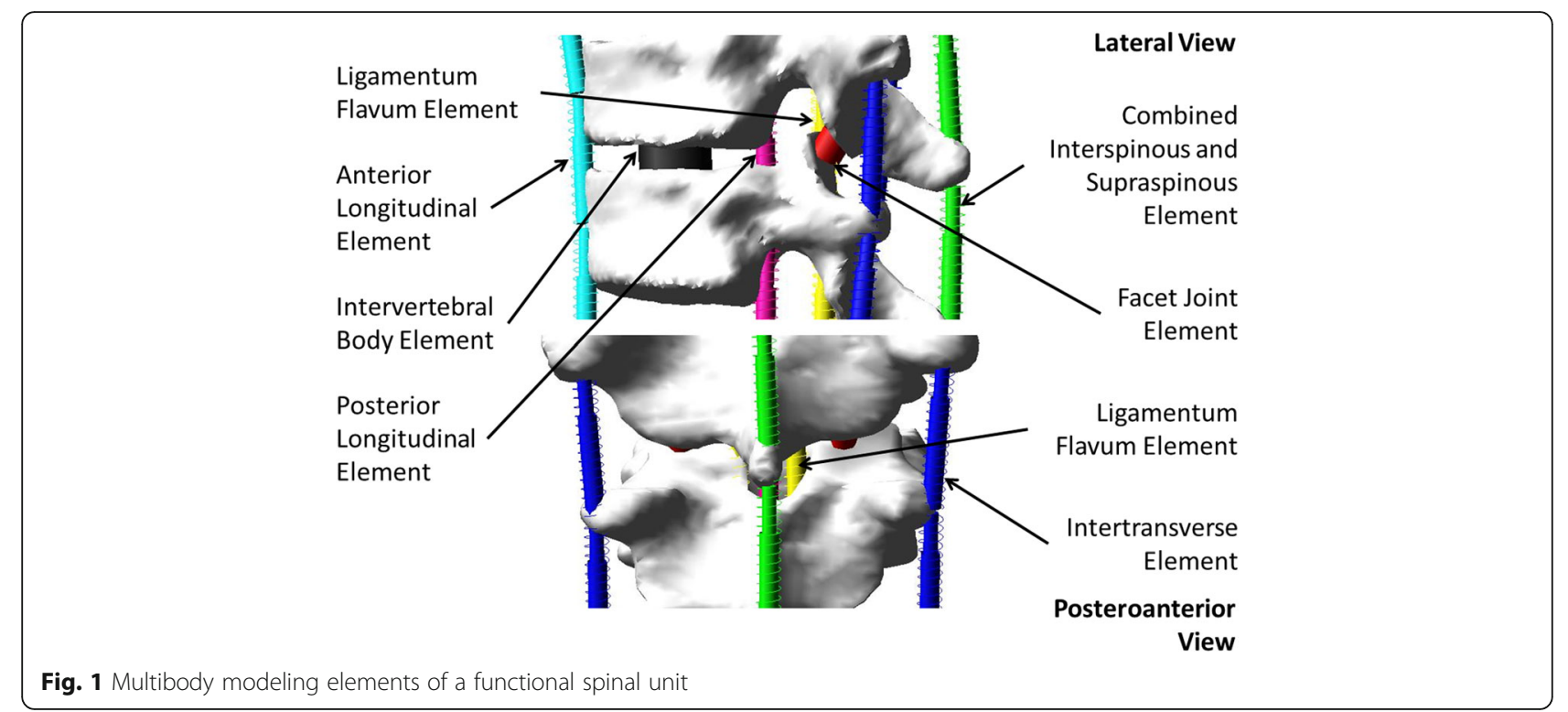

fused and four alternative patterns with mean 23\% fewer screws (21 to 25\%). Screw dropouts in the alternative patterns were respectively on the convex and concave sides and at alternate levels or periapical levels (Fig. 2). The modeled bone-screw connection and correction technique was based on polyaxial pedicle screws with dorsal height adjustability $(4.5-5.5 \mathrm{~mm}$ diameters for the thoracic spine and 5.5-6.0 mm diameters for the lumbar spine) (Fig. 3) [6]. The screw kinematic design allows the translation of each pedicle screw toward the rod from any distance and at any angle, with the ability to rigidly lock the construct at any point between partial and complete corrections [6]. The simulated correction technique was simultaneous two-rod segmental translation. The biomechanical model of the rods was based on $5.5 \mathrm{~mm}$ Cobalt-chrome rods. The contouring angle of the convex rod was $25^{\circ}$ as measured over the thoracic spinal segment, and the contouring angle of the concave rod was $35^{\circ}$. Modeling of instrumentation constructs, simultaneous two-rod segmental translation and the boundary conditions have been realized and validated in a previous study [6].

\section{Results}

The computed geometric indices from the reconstructed preoperative spine models and the simulated instrumented spine models are presented in Table 2. In the 40 simulations with the alternative screw patterns, the final simulated Cobb angle differences varied between $1^{\circ}$ to $5^{\circ}$ in 39 simulations and $8^{\circ}$ in 1 simulation compared to the reference maximal density screw pattern. Thoracic kyphosis and apical vertebral rotation were within $2^{\circ}$ of the reference screw pattern.

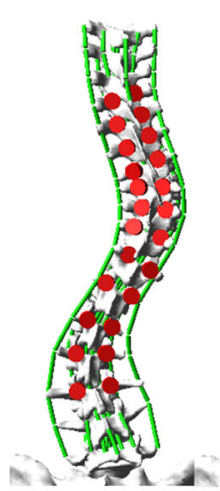

a)

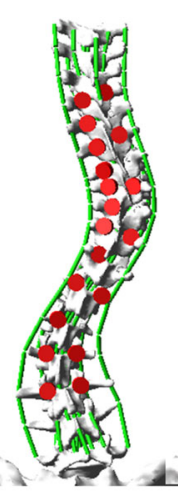

b)

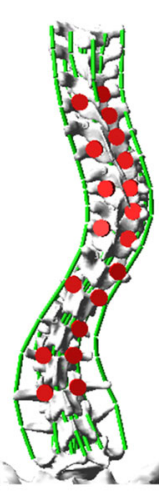

c)

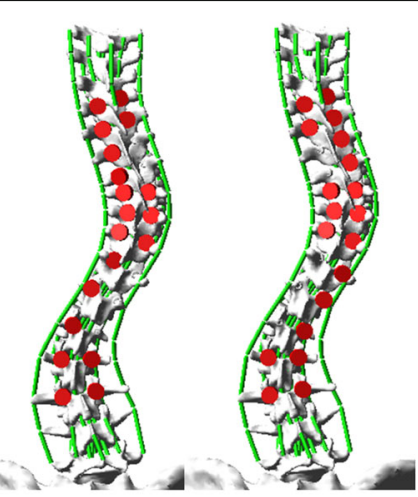

d) e)

Fig. 2 a Reference screw pattern (bilateral screws at every level fused). b Screw pattern with convex alternate screw dropouts. c Concave alternate screw dropouts. d Convex periapical screw dropouts. e Concave periapical screw dropouts 


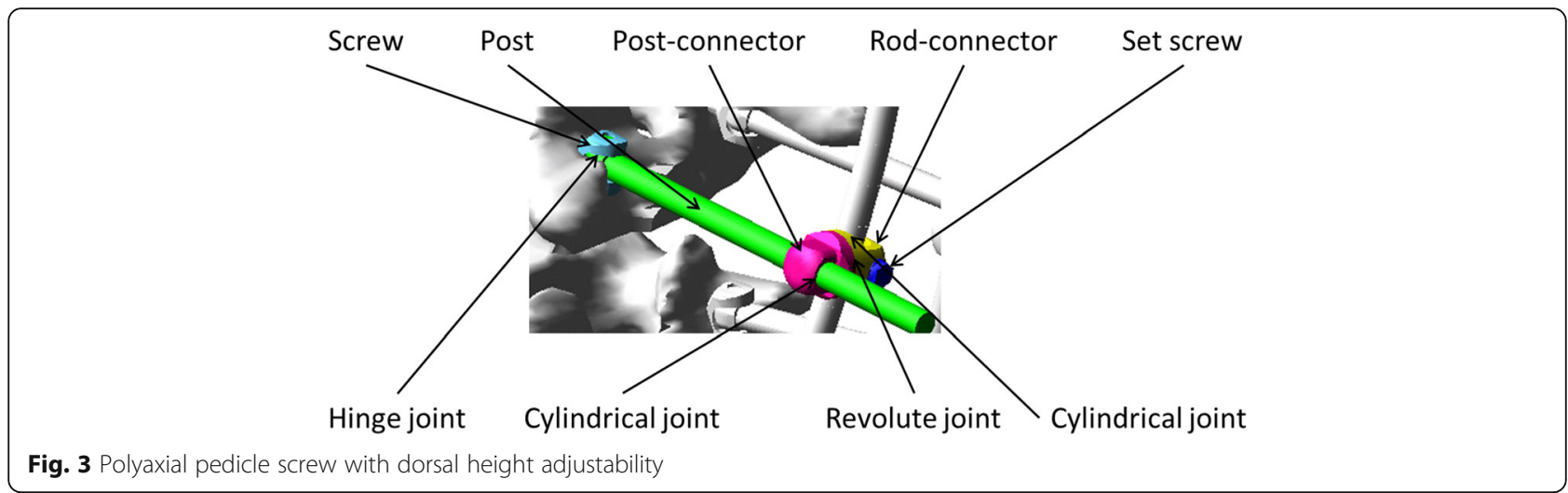

Average bone-screw force was computed for each simulation and the results are presented in Fig. 4. The overall bone-screw force was $76 \pm 43 \mathrm{~N}(5-219 \mathrm{~N})$ for the maximal density reference screw pattern. They were $96 \pm 58 \mathrm{~N}(10-468 \mathrm{~N}), 90 \pm 54 \mathrm{~N}(11-353 \mathrm{~N}), 82 \pm 33 \mathrm{~N}$ $(17-162 \mathrm{~N})$, and $79 \pm 42 \mathrm{~N}(7-222 \mathrm{~N})$, respectively, for the four alternative screw patterns with screw dropouts at convex alternate levels, concave alternate levels, convex periapical levels, and concave periapical levels, which were respectively $26,17,7$, and $4 \%$ higher than the reference screw pattern. Bone-screw forces for the alternative patterns were statistically higher than the reference pattern $(p<0.0003)$. Alternate dropout screw forces were higher than periapical dropouts $(p<0.05)$. There was no statistical bone-screw force difference between convex and concave alternate dropouts $(p>0.28)$. Although there was no statistical bone-screw force difference between convex and concave periapical dropouts $(p>0.25)$, the convex periapical screw dropouts had less impact on bone-screw force vector pattern in some

Table 2 Computed geometric indices from the reconstructed preoperative spine models and the simulated instrumented spine models

\begin{tabular}{|c|c|c|c|c|c|c|c|c|c|c|}
\hline Case no. & 1 & 2 & 3 & 4 & 5 & 6 & 7 & 8 & 9 & 10 \\
\hline \multicolumn{11}{|l|}{ Main thoracic Cobb angles } \\
\hline Preop. & $55^{\circ}$ & $52^{\circ}$ & $58^{\circ}$ & $60^{\circ}$ & $64^{\circ}$ & $62^{\circ}$ & $44^{\circ}$ & $48^{\circ}$ & $67^{\circ}$ & $51^{\circ}$ \\
\hline Pattern no. 1 (reference) & $18^{\circ}$ & $10^{\circ}$ & $18^{\circ}$ & $14^{\circ}$ & $24^{\circ}$ & $21^{\circ}$ & $17^{\circ}$ & $15^{\circ}$ & $28^{\circ}$ & $17^{\circ}$ \\
\hline Pattern no. 2 & $18^{\circ}$ & $12^{\circ}$ & $22^{\circ}$ & $15^{\circ}$ & $25^{\circ}$ & $21^{\circ}$ & $17^{\circ}$ & $16^{\circ}$ & $29^{\circ}$ & $17^{\circ}$ \\
\hline Pattern no. 3 & $18^{\circ}$ & $10^{\circ}$ & $17^{\circ}$ & $22^{\circ}$ & $24^{\circ}$ & $21^{\circ}$ & $15^{\circ}$ & $16^{\circ}$ & $29^{\circ}$ & $17^{\circ}$ \\
\hline Pattern no. 4 & $16^{\circ}$ & $12^{\circ}$ & $20^{\circ}$ & $14^{\circ}$ & $24^{\circ}$ & $22^{\circ}$ & $16^{\circ}$ & $15^{\circ}$ & $30^{\circ}$ & $17^{\circ}$ \\
\hline Pattern no. 5 & $21^{\circ}$ & $9^{\circ}$ & $19^{\circ}$ & $19^{\circ}$ & $24^{\circ}$ & $21^{\circ}$ & $16^{\circ}$ & $15^{\circ}$ & $30^{\circ}$ & $17^{\circ}$ \\
\hline \multicolumn{11}{|l|}{ Thoracic kyphosis } \\
\hline Preop. & $7^{\circ}$ & $23^{\circ}$ & $37^{\circ}$ & $28^{\circ}$ & $22^{\circ}$ & $11^{\circ}$ & $20^{\circ}$ & $18^{\circ}$ & $7^{\circ}$ & $15^{\circ}$ \\
\hline Pattern no. 1 (reference) & $27^{\circ}$ & $31^{\circ}$ & $33^{\circ}$ & $22^{\circ}$ & $24^{\circ}$ & $28^{\circ}$ & $29^{\circ}$ & $27^{\circ}$ & $24^{\circ}$ & $25^{\circ}$ \\
\hline Pattern no. 2 & $26^{\circ}$ & $30^{\circ}$ & $33^{\circ}$ & $20^{\circ}$ & $24^{\circ}$ & $28^{\circ}$ & $29^{\circ}$ & $27^{\circ}$ & $24^{\circ}$ & $25^{\circ}$ \\
\hline Pattern no. 3 & $27^{\circ}$ & $32^{\circ}$ & $33^{\circ}$ & $21^{\circ}$ & $23^{\circ}$ & $28^{\circ}$ & $29^{\circ}$ & $27^{\circ}$ & $24^{\circ}$ & $25^{\circ}$ \\
\hline Pattern no. 4 & $28^{\circ}$ & $31^{\circ}$ & $32^{\circ}$ & $22^{\circ}$ & $23^{\circ}$ & $28^{\circ}$ & $29^{\circ}$ & $27^{\circ}$ & $24^{\circ}$ & $25^{\circ}$ \\
\hline Pattern no. 5 & $26^{\circ}$ & $31^{\circ}$ & $33^{\circ}$ & $21^{\circ}$ & $24^{\circ}$ & $29^{\circ}$ & $29^{\circ}$ & $27^{\circ}$ & $24^{\circ}$ & $25^{\circ}$ \\
\hline \multicolumn{11}{|c|}{ Main thoracic apical vertebral rotation } \\
\hline Preop. & $16^{\circ}$ & $17^{\circ}$ & $18^{\circ}$ & $19^{\circ}$ & $19^{\circ}$ & $20^{\circ}$ & $19^{\circ}$ & $18^{\circ}$ & $22^{\circ}$ & $19^{\circ}$ \\
\hline Pattern no. 1 (reference) & $15^{\circ}$ & $18^{\circ}$ & $18^{\circ}$ & $17^{\circ}$ & $17^{\circ}$ & $19^{\circ}$ & $18^{\circ}$ & $17^{\circ}$ & $20^{\circ}$ & $18^{\circ}$ \\
\hline Pattern no. 2 & $14^{\circ}$ & $18^{\circ}$ & $17^{\circ}$ & $16^{\circ}$ & $17^{\circ}$ & $19^{\circ}$ & $19^{\circ}$ & $17^{\circ}$ & $21^{\circ}$ & $19^{\circ}$ \\
\hline Pattern no. 3 & $15^{\circ}$ & $18^{\circ}$ & $18^{\circ}$ & $15^{\circ}$ & $17^{\circ}$ & $19^{\circ}$ & $17^{\circ}$ & $17^{\circ}$ & $20^{\circ}$ & $18^{\circ}$ \\
\hline Pattern no. 4 & $15^{\circ}$ & $18^{\circ}$ & $18^{\circ}$ & $17^{\circ}$ & $16^{\circ}$ & $19^{\circ}$ & $18^{\circ}$ & $17^{\circ}$ & $21^{\circ}$ & $19^{\circ}$ \\
\hline Pattern no. 5 & $14^{\circ}$ & $18^{\circ}$ & $19^{\circ}$ & $15^{\circ}$ & $17^{\circ}$ & $19^{\circ}$ & $17^{\circ}$ & $17^{\circ}$ & $20^{\circ}$ & $18^{\circ}$ \\
\hline
\end{tabular}

Pattern no. 1 (reference): bilateral screws at every level fused; pattern no. 2: convex alternate screw dropouts; pattern no. 3: concave alternate screw dropouts; pattern no. 4: convex periapical screw dropouts; pattern no. 5: concave periapical screw dropouts 


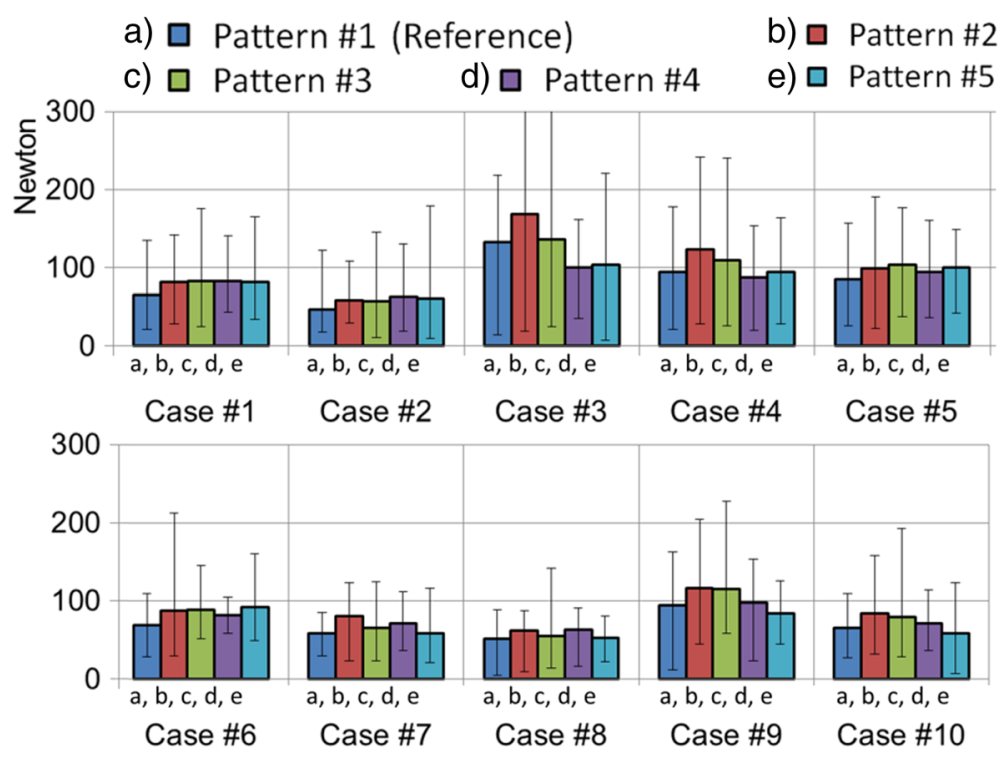

Fig. 4 Average (bars = min, max) bone-screw forces (pattern no. 1 (reference): bilateral screws at every level fused; pattern no. 2: convex alternate screw dropouts; pattern no. 3: concave alternate screw dropouts; pattern no. 4: convex periapical screw dropouts; pattern no. 5: concave periapical screw dropouts)

of the cases in both the coronal and the sagittal plane, i.e., bone-screw force vector pattern was the closest to the reference screw pattern. Bone-screw force vectors for a representative case are provided in Fig. 5.

\section{Discussion}

Comparing alternative screw patterns respectively with the maximal density reference screw pattern, differences in the final simulated MT Cobb angles, thoracic kyphosis, and apical vertebral rotation did not exceed $5^{\circ}$ for all except one case. These differences are within the accepted systematic error found in clinical Cobb angle measurements [40]. The mean correction of each of the alternative screw pattern was within $2^{\circ}$ of the mean correction of the reference screw pattern. Based on a previous study on 279 AIS patients [9] and a structured literature review on AIS instrumentations [7], the population mean of major curve Cobb angles was estimated to be $55^{\circ}$ and the population mean of percent corrections of major curves was estimated to be $67 \%$ with a standard deviation of $14 \%$. The correction difference to be detected was set to $5^{\circ}$ (11\% difference in percent correction). There was no statistical difference between the reference screw pattern and the alternative screw patterns in terms of percent corrections of major curves, with $5 \%$ of type I error and a statistical power of $70 \%$.

Alternative screw patterns with fewer screws resulted in higher overall bone-screw forces. Previous studies showed that higher density screw patterns had usually higher bone-screw forces due to the overconstraining effect [41]. The difference can be attributed to differences between the construct designs, type of screws, and simulated correction techniques. Higher bone-screw forces were generally associated with higher Cobb angles when percent corrections were similar; bone-screw forces in curves of higher Cobb angles may be more sensitive to screw density and distribution. The number of vertebrae in the major curve and the local shape of the curve seem to have an important effect on the average bone-screw force; higher forces were more seen in cases in which the major curves spanned fewer vertebrae and were more angular (Fig. 6). In other words, curves which span longer spinal segment and whose curvature does not varies significantly tend to have lower bone-screw forces. A sharp, short angular curve with a high local Cobb angle requires greater corrective forces to align the spine to the smoothly contoured spinal rods. Short, angular curves may therefore be more sensitive to screw density and distribution, which should be taken into consideration in addition to curve flexibility. Significantly higher bone-screw forces in some cases may be explained by the fact that the curve spanned a shorter spinal segment and was more angular (cases 3, 4, and 9). In the sagittal plane, the spinal profiles in some cases may have a better match with the rod shapes than in other cases, which should also have an impact on the final bone-screw forces. Screw density and distribution should therefore be determined by taking into account the local geometric characteristics of the curve in both the sagittal and coronal planes in addition to the curve type, deformity magnitude, and spinal stiffness.

In summary, with simultaneous two-rod segmental translation using polyaxial pedicle screws with dorsal 


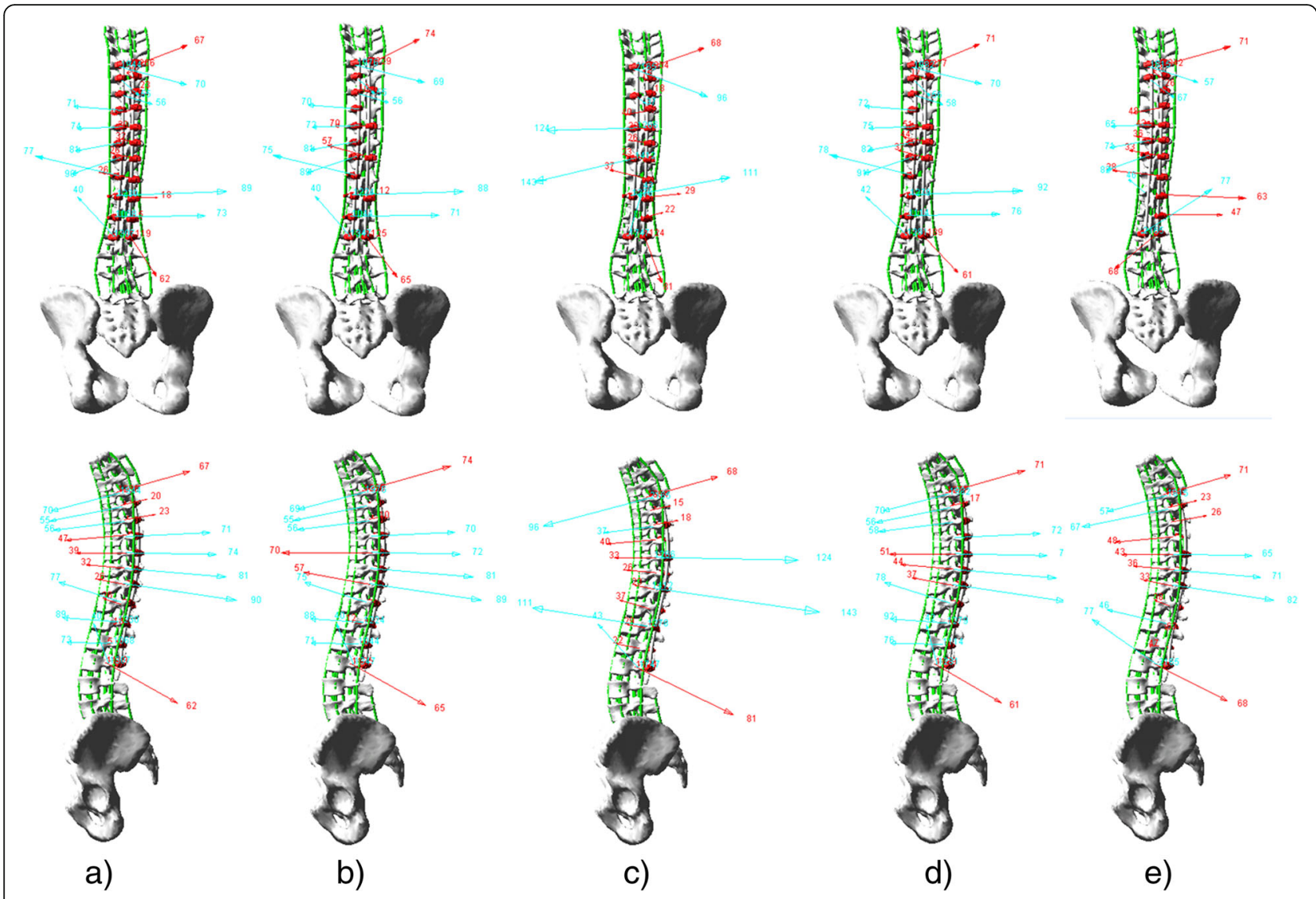

Fig. 5 Sample bone-screw force patterns (case no. 8) with (a) the reference screw pattern (bilateral screws at every level fused) and screw patterns with (b) convex alternate screw dropouts, (c) concave alternate screw dropouts, (d) convex periapical screw dropouts, and (e) concave periapical screw dropouts (red arrows: convex side bone-screw force vectors; cyan arrows: concave side bone-screw force vectors)

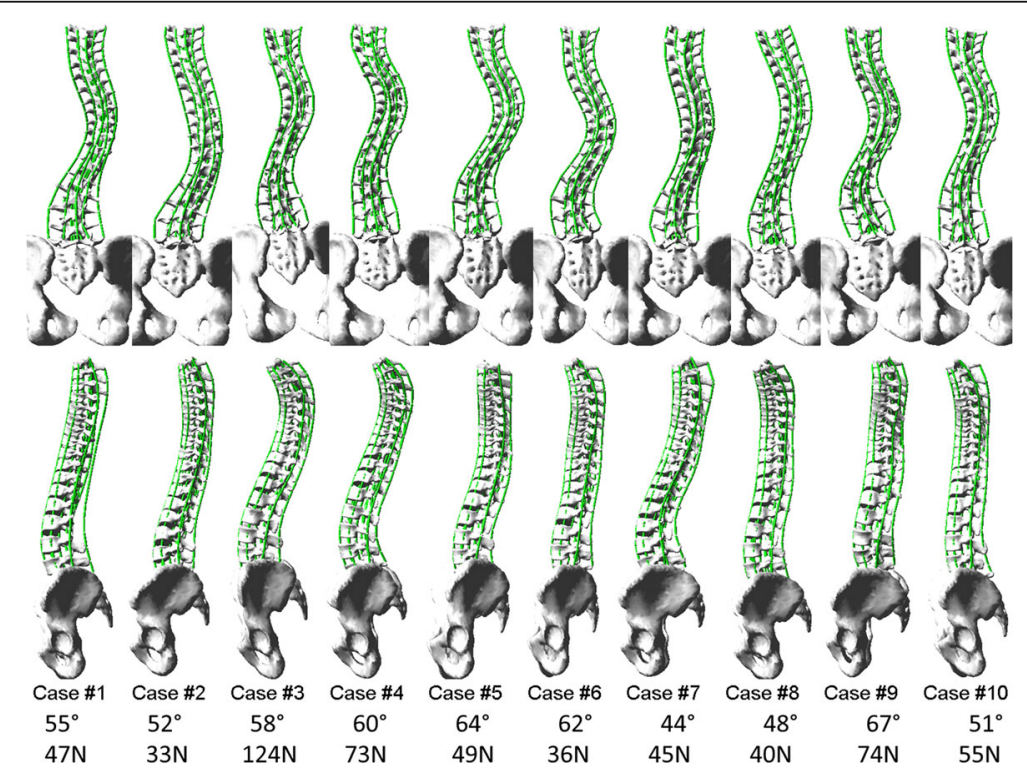

Fig. 6 Posteroanterior and lateral views of the preoperative spine models, major curve Cobb angles and the average bone-screw forces with bilateral screws at each level fused 
height adjustability, there was no statistical difference between convex side screw dropouts and concave side screw dropouts. Periapical screw dropouts may more likely result in lower bone-screw force increase than alternate level screw dropouts. The number of vertebrae spanned by the major curve or the sharpness of the curve affects the bone-screw forces; screw dropouts should be reduced within short structural curves with high local curvature. Screw density and distribution should be determined by taking into account the local geometric characteristics of the curve in both the sagittal and coronal planes and the shape of the rods in addition to the curve type, deformity magnitude, and spinal stiffness.

Different correction techniques and instrumentation construct designs may have important roles in curve correction and bone-screw forces. Since the simulated correction technique was simultaneous two-rod segmental translation using polyaxial pedicle screws with dorsal height adjustability, findings in this study may not be directly applied to other techniques, types of screws, and construct designs. However, based on the fundamental laws of mechanics, for an equivalent curve correction, the overall effective corrective forces should be at an equivalent level independent of correction techniques and construct designs. Findings in this study provided therefore useful data on the overall effect of screw density and distribution. Knowledge of potential bone-screw forces in AIS instrumentation using alternative computersimulated screw constructs can help surgeons select the best possible screw configuration specific for the patient. All bone-screw forces may not contribute to the actual curve correction due to the high mechanic complexity of the instrumented spine. Parts of the bone-screw forces are the "true corrective forces," which are necessary and sufficient to achieve the desired correction and the rest are overconstraining forces which are induced when forcing to ensure proper rod seating and locking at all pedicle screws as required by the construct design [42]. The effects of screw design, and density and distribution on true corrective forces and overconstraining forces need to be investigated with more AIS cases using various correction techniques and construct designs. Depending on the pedicle size of each individual patient, the pedicle screw diameter used varies among patients, which should be an important factor in determining the screw density and distribution and needs to be investigated.

This study is limited by the available experimental data to calibrate and describe the biomechanical properties of the scoliotic spine model. However, the modeling technique has been adapted to make the best of the available calibration data to meet the needs of this study. Some simplifications were made, such as modeling the vertebral bodies as rigid parts, limiting the model solving in the quasistatic domain, and approximating the intervertebral connection with limited number of elastic elements. Since the focus was on the overall comparative curve correction and bonescrew forces, these simplifications and approximations were considered as adequate for this study. To establish baseline data for screw density and distribution, studies through simulations using computerized biomechanical models should be combined with prospective clinical studies and biomechanical experiments. The computerized model will be refined and better calibrated using the more comprehensive clinical and experimental results and then used to perform more extensive studies which may not be possible in a clinical and experimental context.

\section{Conclusions}

Deformity correction can be achieved with $23 \%$ fewer screws than maximal density screw pattern, in which screw dropouts could be either on the convex side or on the concave side at alternate levels or at periapical levels. Using fewer screws resulted in higher average bonescrew forces. Findings in this study provided preliminary data on the effect of screw density and distribution. Further studies should be conducted on more screw densities and distributions using different correction techniques and different instrumentation in order to acquire comprehensive biomechanical knowledge to assist in individualized surgical treatment for AIS.

\section{Abbreviations \\ 3D: Three-dimension; AIS: Adolescent idiopathic scoliosis; ALL: Anterior longitudinal ligament; AVR: Apical vertebral rotation; FC: Facet joint capsule; ISL: Interspinous ligament; ITL: Intertransverse ligament; LF: Ligamentum flavum; LIV: Lower instrumented vertebra; MT: Main thoracic; PLL: Posterior longitudinal ligament; PT: Proximal thoracic; SD: Standard deviation; SSL: Supraspinous ligament; TL/L: Thoracolumbar/lumbar; UIV: Upper instrumented vertebra}

\section{Acknowledgements}

The authors acknowledge the Natural Sciences and Engineering Research Council of Canada (Industrial Research Chair program with Medtronic of Canada) and the Scoliosis Research Society for providing financial supports. The authors acknowledge Julie Joncas for her efforts in the clinical data collection.

\section{Funding}

This study was financially supported by the Natural Sciences and Engineering Research Council of Canada (Industrial Research Chair program with Medtronic of Canada) and the Scoliosis Research Society.

\section{Availability of data and materials Not applicable.}

\section{Authors' contributions \\ XW and C-EA performed the study design, data collection and compilation, biomechanical modeling and simulations, result analysis, and manuscript drafting and revision. ANL and DGC all made substantial contributions to the study design, result analysis, and manuscript revision. SP, HL, and CGTL all made substantial contributions to the data collection, results analysis, and manuscript revision. All authors read and approved the final manuscript.}

Competing interests

The authors declare that they have no competing interests. 


\section{Consent for publication}

Not applicable.

\section{Ethics approval and consent to participate}

The study was performed within the framework of a research project which is being conducted with the approval of the Institutional Review Board (Comite d'ethique de la recherche) of Sainte-Justine University Hospital Center. The title of the research project is "Modeling and simulation of scoliosis instrumentation surgery" (Modelisation et simulation de la chirurgie d'instrumentation du rachis scoliotique); the reference number of the approval is \#2668.

\section{Publisher's Note}

Springer Nature remains neutral with regard to jurisdictional claims in published maps and institutional affiliations.

\section{Author details}

${ }^{1}$ Department of Mechanical Engineering, Polytechnique Montréal, P.O. Box 6079, Downtown Station, Montreal, Quebec H3C 3A7, Canada. ${ }^{2}$ Sainte-Justine University Hospital Center, 3175, Cote Sainte-Catherine Road, Montreal, Quebec H3T 1C5, Canada. ${ }^{3}$ Department of Orthopedic Surgery, Mayo Clinic, 2001 st Street SW, Rochester, MN 55905, USA. ${ }^{4}$ Sonoran Spine Center and Research Foundation, 1255 W Rio Salado Pkwy, Suite 107, Tempe, AZ 85281 USA. ${ }^{5}$ Department of Orthopaedic Surgery, University of Minnesota, 2450 Riverside Avenue South, Suite R200, Minneapolis, MN 55454, USA

\section{Received: 1 July 2016 Accepted: 28 March 2017}

Published online: 17 April 2017

\section{References}

1. Ledonio CG, Polly Jr DW, Vitale MG, et al. Pediatric pedicle screws: comparative effectiveness and safety: a systematic literature review from the Scoliosis Research Society and the Pediatric Orthopaedic Society of North America task force. J Bone Joint Surg Am. 2011;93:1227-34.

2. Lenke LG, Kuklo TR, Ondra S, et al. Rationale behind the current state-of-theart treatment of scoliosis (in the pedicle screw era). Spine (Phila Pa 1976). 2008;33:1051-4

3. Crawford AH, Lykissas MG, Gao X, et al. All-pedicle screw versus hybrid instrumentation in adolescent idiopathic scoliosis surgery: a comparative radiographical study with a minimum 2-year follow-up. Spine (Phila Pa 1976). 2013:38:1199-208.

4. Le Naveaux F, Aubin CE, Larson AN, et al. Key anchor points for specific correction maneuvers in Lenke 1 AIS: how important is the implant pattern design? In SRS ed. The 22nd International Meeting on Advanced Spine Techniques (IMAST). Kuala Lumpur, Malaysia, 2015.

5. Wang $X$, Aubin CE, Crandall D, et al. Biomechanical analysis of 4 types of pedicle screws for scoliotic spine instrumentation. Spine (Phila Pa 1976). 2012;37:E823-35.

6. Wang $X$, Aubin CE, Crandall D, et al. Biomechanical modeling and analysis of a direct incremental segmental translation system for the instrumentation of scoliotic deformities. Clin Biomech (Bristol, Avon) 2011:26:548-55.

7. Larson AN, Aubin CE, Polly Jr DW, et al. Are more screws better? A systematic review of anchor density and curve correction in adolescent idiopathic scoliosis. Spine Deformity. 2013;1:237-47.

8. Larson AN, Polly Jr DW, Diamond B, et al. Does higher anchor density result in increased curve correction and improved clinical outcomes in adolescent idiopathic scoliosis? Spine (Phila Pa 1976). 2014;39:571-8.

9. Le Naveaux F, Aubin CE, Larson AN, et al. Implant distribution in surgically instrumented Lenke 1 adolescent idiopathic scoliosis: does it affect curve correction? Spine (Phila Pa 1976). 2015;40:462-8.

10. Bharucha NJ, Lonner BS, Auerbach JD, et al. Low-density versus high-density thoracic pedicle screw constructs in adolescent idiopathic scoliosis: do more screws lead to a better outcome? Spine J. 2013:13:375-81.

11. Di Silvestre M, Parisini P, Lolli F, et al. Complications of thoracic pedicle screws in scoliosis treatment. Spine (Phila Pa 1976). 2007:32:1655-61.

12. Mac-Thiong JM, Parent S, Poitras B, et al. Neurological outcome and management of pedicle screws misplaced totally within the spinal canal. Spine (Phila Pa 1976). 2013;38:229-37.

13. Sugarman $E$, Sarwahi $V$, Amaral $T$, et al. Comparative analysis of perioperative differences between hybrid versus pedicle screw instrumentation in adolescent idiopathic scoliosis. J Spinal Disord Tech. 2013:26:161-6.

14. Ul Haque M, Shufflebarger HL, O'Brien M, et al. Radiation exposure during pedicle screw placement in adolescent idiopathic scoliosis: is fluoroscopy safe? Spine (Phila Pa 1976). 2006;31:2516-20.

15. Chen J, Yang C, Ran B, et al. Correction of Lenke 5 adolescent idiopathic scoliosis using pedicle screw instrumentation: does implant density influence the correction? Spine (Phila Pa 1976). 2013;38:E946-51.

16. Gotfryd $A O$, Avanzi $O$. Randomized clinical study on surgical techniques with different pedicle screw densities in the treatment of adolescent idiopathic scoliosis types Lenke 1A and 1B. Spine Deformity. 2013;1:272-9.

17. Cheriet F, Laporte C, Kadoury S, et al. A novel system for thE 3-D reconstruction of the human spine and rib cage from biplanar X-ray images. IEEE Trans Biomed Eng. 2007;54:1356-8.

18. Delorme S, Petit $Y$, de Guise JA, et al. Assessment of the 3-D reconstruction and high-resolution geometrical modeling of the human skeletal trunk from 2-D radiographic images. IEEE Trans Biomed Eng. 2003:50:989-98.

19. Jaumard NV, Welch WC, Winkelstein BA. Spinal facet joint biomechanics and mechanotransduction in normal, injury and degenerative conditions. J Biomech Eng. 2011;133:071010.

20. Watkins $R$, Watkins 3rd R, Williams $L$, et al. Stability provided by the sternum and rib cage in the thoracic spine. Spine (Phila Pa 1976). 2005;30:1283-6.

21. Myklebust JB, Pintar F, Yoganandan N, et al. Tensile strength of spinal ligaments. Spine (Phila Pa 1976). 1988;13:526-31.

22. Pintar FA. The biomechanics of spinal elements (ligaments, vertebral body, disc). Ann Arbor: Marquette University; 1986. p. 237.

23. Tong SY-P. A mechanical model of the normal human spine. Ann Arbor: University of Alberta (Canada): 1999. p. 164.

24. Holewijn RM, Schlösser TPC, Bisschop A, et al. How does spinal release and Ponte osteotomy improve spinal flexibility? The law of diminishing returns. Spine Deformity. 2015;3:489-95.

25. Wang C, Bell K, McClincy M, et al. Biomechanical comparison of Ponte osteotomy and discectomy. Spine (Phila Pa 1976). 2015;40:E141-5.

26. Wiemann J, Durrani S, Bosch P. The effect of posterior spinal releases on axial correction torque: a cadaver study. J Child Orthop. 2011;5:109-13.

27. Wollowick AL, Farrelly EE, Meyers K, et al. Anterior release generates more thoracic rotation than posterior osteotomy: a biomechanical study of human cadaver spines. Spine (Phila Pa 1976). 2013;38:1540-5.

28. Anderson AL, Mclff TE, Asher MA, et al. The effect of posterior thoracic spine anatomical structures on motion segment flexion stiffness. Spine (Phila Pa 1976). 2009:34:441-6.

29. Panjabi MM, Hausfeld JN, White 3rd AA. A biomechanical study of the ligamentous stability of the thoracic spine in man. Acta Orthop Scand. 1981:52:315-26

30. Adams MA, Hutton WC. The effect of posture on the role of the apophysial joints in resisting intervertebral compressive forces. J Bone Joint Surg (Br). 1980;62:358-62.

31. Pal GP, Routal RV. A study of weight transmission through the cervical and upper thoracic regions of the vertebral column in man. J Anat. 1986;148:245-61.

32. Pal GP, Routal RV. Transmission of weight through the lower thoracic and lumbar regions of the vertebral column in man. J Anat. 1987;152:93-105.

33. Yang KH, King Al. Mechanism of facet load transmission as a hypothesis for low-back pain. Spine (Phila Pa 1976). 1984;9:557-65.

34. Gardner-Morse MG, Stokes IA. Structural behavior of human lumbar spinal motion segments. J Biomech. 2004;37:205-12.

35. Panjabi MM, Brand Jr RA, White 3rd AA. Three-dimensional flexibility and stiffness properties of the human thoracic spine. J Biomech. 1976;9:185-92.

36. Panjabi MM, Brand Jr RA, White 3rd AA. Mechanical properties of the human thoracic spine as shown by three-dimensional load-displacement curves. J Bone Joint Surg Am. 1976;58:642-52.

37. Panjabi MM, Oxland TR, Yamamoto I, et al. Mechanical behavior of the human lumbar and lumbosacral spine as shown by three-dimensional loaddisplacement curves. J Bone Joint Surg Am. 1994;76:413-24.

38. Aubin CE, Labelle $H$, Chevrefils $C$, et al. Preoperative planning simulator for spinal deformity surgeries. Spine (Phila Pa 1976). 2008;33:2143-52.

39. Petit $Y$, Aubin $C E$, Labelle H. Patient-specific mechanical properties of a flexible multi-body model of the scoliotic spine. Med Biol Eng Comput. 2004;42:55-60.

40. Dang NR, Moreau MJ, Hill DL, et al. Intra-observer reproducibility and interobserver reliability of the radiographic parameters in the Spinal 
Deformity Study Group's AIS Radiographic Measurement Manual. Spine (Phila Pa 1976). 2005;30:1064-9.

41. Wang X, Aubin CE, Robitaille I, et al. Biomechanical comparison of alternative densities of pedicle screws for the treatment of adolescent idiopathic scoliosis. Eur Spine J. 2012;21:1082-90.

42. Wang $X$, Aubin $C E$, Labelle $H$, et al. Biomechanical analysis of corrective forces in spinal instrumentation for scoliosis treatment. Spine (Phila Pa 1976). 2012;37:E1479-87.

Submit your next manuscript to BioMed Central and we will help you at every step:

- We accept pre-submission inquiries

- Our selector tool helps you to find the most relevant journal

- We provide round the clock customer support

- Convenient online submission

- Thorough peer review

- Inclusion in PubMed and all major indexing services

- Maximum visibility for your research

Submit your manuscript at www.biomedcentral.com/submit 